Pacific Journal of Mathematics

SOLUTIONS OF DIFFERENTIAL EQUATIONS WITH

CH. G. PH LOS AND V. A. STAÏKOS 


\title{
ASYMPTOTIC PROPERTIES OF NONOSCILLATORY SOLUTIONS OF DIFFERENTIAL EQUATIONS WITH DEVIATING ARGUMENT
}

\author{
Ch. G. Philos and V. A. Staikos
}

Recently, there is an increasing interest in studying the $n$th order differential equations involving the so called $n$th order $r$-derivative of $x$

$$
\left(r_{n-1}(t)\left(r_{n-2}(t)\left(\cdots\left(r_{1}(t)\left(r_{0}(t) x(t)\right)^{\prime}\right)^{\prime} \cdots\right)^{\prime}\right)^{\prime}\right)^{\prime}
$$

which causes damped terms. Here, the asymptotic behavior of nonoscillatory solutions of such general differential equations with deviating argument is studied and, more precisely, sufficient conditions which guarantee that

$$
\lim _{t \rightarrow \infty} x(t)=0
$$

for the bounded nonoscillatory solutions $x(t)$ are established. A basic theorem is obtained for the general case and then it is specialized into four corollaries concerning the particular case

$$
r_{\jmath}=1 \text { for } j \neq n-N \text { and } r_{n-N}=r \quad(1 \leqq N \leqq n-1)
$$

which is of special interest. Finally, some examples are given to illustrate the significance of the results.

In this paper we consider the $n$th order $(n>1)$ differential equation with deviating argument of the form

$$
\begin{gathered}
\left(r_{n-1}(t)\left(r_{n-2}(t)\left(\cdots\left(r_{1}(t)\left(r_{0}(t) x(t)\right)^{\prime}\right)^{\prime} \cdots\right)^{\prime}\right)^{\prime}\right)^{\prime} \\
+a(t) F(x[\sigma(t)])=b(t), \quad t \geqq t_{0}
\end{gathered}
$$

where the functions $r_{i}(i=0,1, \cdots, n-1)$ are supposed positive at least on the interval $\left[t_{0}, \infty\right)$. The continuity of the functions involved in the above equation (E) as well as sufficient smoothness to guarantee the existence of solutions of (E) on an infinite subinterval of $\left[t_{0}, \infty\right)$ will be assumed without mention. In what follows the term "solution" is always used only for such solutions $x(t)$ of $(\mathrm{E})$ which are defined for all large $t$. The oscillatory character is considered in the usual sense, i.e. a continuous real-valued function which is defined on an interval of the form $[T, \infty)$ 
is called oscillatory if it has no last zero, and otherwise it is called nonoscillatory.

Furthermore, the conditions (i) and (ii) below are assumed to hold throughout the paper:

(i) $\lim _{t \rightarrow \infty} \sigma(t)=\infty$

(ii) $y \neq 0 \Rightarrow y F(y)>0$.

The results of this paper are included in a general theorem given in $\S 2$, which establishes sufficient conditions in order that all bounded nonoscillatory solutions $x(t)$ of the differential equation $(E)$ tend to zero as $t \rightarrow \infty$. This theorem extends a recent result given by the authors in [9, Theorem 3] concerning also the equation (E). Also, it unifies and extends two results by Kusano and Onose [5, Theorems 3 and 4] concerning, in particular, the differential equation

$\left(\mathrm{D}_{N}\right) \quad\left[r(t) x^{(n-N)}(t)\right]^{(N)}+a(t) F(x[\sigma(t)])=b(t), \quad t \geqq t_{0}$,

where $1 \leqq N \leqq n-1$ and the function $r$ is continuous and positive at least on the interval $\left[t_{0}, \infty\right)$.

The technique used in the proof of our theorem is based on three lemmas which are given in $\S 1$. Lemma 1 is fundamental and has been proved by the authors in [9], Lemma 2 is proved here and it is an extension of a result due to Hardy and Littlewood [4], while Lemma 3 is new and it is rather technical.

In $\$ 3$ our main theorem is specialized for the differential equation $\left(D_{N}\right)$, which is obtained from the equation (E) by setting

$$
r_{j}=1 \text { for } j \neq n-N \text { and } r_{n-N}=r
$$

and which is of special interest. Thus, four corollaries are obtained, from which Corollary 1 is the main result of a recent paper by Kusano and Onose [5] while Corollaries 2, 3 and 4 are new and are illustrated by examples.

1. Preliminaries. Let $q_{i}(i=0,1, \cdots, m)$ be positive continuous functions on an interval $I$ of the real line. For a real-valued function $h$ on $I$ and any $\mu=0,1, \cdots, m$ we define the $\mu$ th $q$-derivative of $h$ by the formula

$$
D_{q}^{(\mu)} h=q_{\mu}\left(q_{\mu-1}\left(q_{\mu-2}\left(\cdots\left(q_{1}\left(q_{0} h\right)^{\prime}\right)^{\prime} \cdots\right)^{\prime}\right)^{\prime}\right)^{\prime}
$$

when obviously we have

$$
D_{q}^{(0)} h=q_{0} h
$$




$$
D_{q}^{(1)} h=q_{j}\left(D_{q}^{(1-1)} h\right)^{\prime} \quad(j=1,2, \cdots, m) .
$$

Moreover, if $D_{q}^{(m)} h$ is defined as a continuous function on $I$, then $h$ is said to be $m$-times continuously $q$-differentiable. We note that in the case where $q_{0}=q_{1}=\cdots=q_{m}=1$ the above notion of $q$-differentiability specializes to the usual one.

By using this shorthand notation, the differential equation $(E)$ can be written

$$
\left(D_{r}^{(n)} x\right)(t)+a(t) F(x[\sigma(t)])=b(t), \quad t \geqq t_{0}
$$

where $r_{n}=1$.

Now, let $\rho$ be a real-valued function which is defined and positive at least on the interval $\left(t_{0}, \infty\right)$ and let $R_{t}(i=0,1, \cdots, n)$ be the functions defined as follows:

$$
R_{n}=\rho
$$

and for every $j=n-1, n-2, \cdots, 0$

$$
R_{\jmath}=r_{\jmath} R_{j+1}^{\prime}
$$

The function $\rho$ is said to be of the type $r[k], 0 \leqq k \leqq n-1$, if:

$(\alpha)$ the functions $R_{j}(j=k+1, \cdots, n)$ are defined at least on $\left(t_{0}, \infty\right)$

( $\beta) \quad R_{k+1}$ is a constant nonzero function on $\left(t_{0}, \infty\right)$,

$(\gamma)$ if $k<n-1$, then for every $j=k+2, \cdots, n$

$$
\lim _{t \rightarrow \infty} R_{l}(t) \text { exists in }\{-\infty, 0,+\infty\}
$$

and

( $\delta$ ) if $k<n-2$, then for every $j=k+2, \cdots, n-1$

$$
R_{,}(t) \neq 0 \text { for all } t>t_{0} .
$$

For some interesting examples of functions of the above type we refer to [9].

The technique used here is based on the following lemma, which has been proved by the authors in [9].

LEMMA 1. Let $\rho$ be a function of the type $r[k], 0 \leqq k \leqq n-1$, and $h$ an $n$-times continuously $r$-differentiable function of $[T, \infty), T>t_{0}$.

If the improper integral 


$$
\int_{T}^{\infty} \rho(t)\left(D_{r}^{(n)} h\right)(t) d t
$$

exists in the extended real line $\mathbf{R}^{*}$, then so does the $\lim _{t \rightarrow \infty}\left(D_{r}^{(k)} h\right)(t)$. Moreover,

$$
\int_{T}^{\infty} \rho(t)\left(D_{r}^{(n)} h\right)(t) d t= \pm \infty \quad \text { implies } \quad \lim _{t \rightarrow \infty}\left|\left(D_{r}^{(k)} h\right)(t)\right|=\infty
$$

In order to obtain our results we need further the following lemma which is an extension of a result due to Hardy and Littlewood [4].

LeMmA 2. Let $q_{i}(i=0,1, \cdots, m)$, where $m>1$, be positive continuous functions on an interval $[T, \infty)$ such that

$$
\liminf _{t \rightarrow \infty} q_{i}(t)>0 \quad(i=1,2, \cdots, m)
$$

and

$$
\limsup _{t \rightarrow \infty} q_{i}(t)<\infty \quad(i=1,2, \cdots, m-1) .
$$

Moreover, let $h$ be an $m$-times continuously $q$-differentiable function on $[T, \infty)$.

If $D_{q}^{(0)} h$ is bounded on $[T, \infty)$ and

$$
\lim _{t \rightarrow \infty}\left(D_{q}^{(m)} h\right)(t)=0
$$

then

$$
\lim _{t \rightarrow \infty}\left(D_{q}^{(j)} h\right)(t)=0 \quad(j=1,2, \cdots, m-1) .
$$

The above lemma follows immediately from the following proposition, which in the particular case $q_{0}=q_{1}=\cdots=q_{m}=1$ is an improved version of a result due to Landau [6] (cf. also Coppel [2, p. 140]).

Proposition. Let $q_{i}(i=0,1, \cdots, m)$, where $m>1$, be positive continuous functions on an interval I such that

$$
A \equiv \min _{i=1, \cdots, m} \inf _{t \in I} q_{i}(t)>0 \quad \text { and } \quad B \equiv \max _{i=1, \cdots, m-1} \sup _{t \in I} q_{i}(t)<\infty \text {. }
$$


Moreover, let $h$ be an $m$-times continuously $q$-differentiable function on I with

$$
\left|\left(D_{q}^{(0)} h\right)(t)\right| \leqq K \quad \text { and } \quad\left|\left(D_{q}^{(m)} h\right)(t)\right| \leqq M \quad \text { for every } t \in I .
$$

If $L$ is the length of the interval I and

(a) $I$ is closed and $L \geqq 2 A\left(\frac{K}{M}\right)^{1 / m}$

or

(b) $L>2 A\left(\frac{K}{M}\right)^{1 / m}$,

then for every $t \in I$

$$
\left|\left(D_{q}^{(j)} h\right)(t)\right| \leqq c_{m} K^{1-\jmath / m} M^{\prime / m} \quad(j=1,2, \cdots, m-1),
$$

where

$$
c_{m} \equiv\left(\frac{2 B}{A}\right)^{2^{m-2}}
$$

Proof. It suffices to prove the proposition in the case where (a) is satisfied. Indeed, if (b) holds, then for any $t \in I$ we can choose a closed subinterval $J$ of $I$ with length $L^{\prime} \geqq 2 A(K / M)^{1 / m}$ and $t \in J$. So, applying the proposition for the closed interval $J$ we obviously obtain

$$
\left|\left(D_{q}^{(j)} h\right)(t)\right| \leqq c_{m} K^{1-\jmath / m} M^{\jmath / m} \quad(j=1,2, \cdots, m-1) .
$$

Now, we suppose that (a) is satisfied and define

$$
S=\max \left\{c_{m}, \max _{0<j<m} \max _{t \in I} \frac{\left|\left(D_{q}^{(j)} h\right)(t)\right|}{K^{1-j / m} M^{j / m}}\right\} .
$$

Obviously, for every $j=0,1, \cdots, m-1$

$$
1 \leqq\left(\frac{2 B}{A}\right)^{2^{\prime+1}-2} \leqq\left(\frac{2 B}{A}\right)^{2^{m}-2}=c_{m} \leqq S
$$

by which, after some manipulations, we derive that

$$
\left(\frac{4 B^{2}}{A^{2}} S\right)^{\gamma_{j}} \leqq S,
$$

where $\gamma_{\jmath}=1-2^{-\prime}$.

We shall prove next that for $j=0,1, \cdots, m-1$ 
(2)

$$
\left|\left(D_{q}^{(j)} h\right)(t)\right| \leqq\left(\frac{4 B^{2}}{A^{2}} S\right)^{\gamma_{j}} K^{1-j / m} M^{j / m} \equiv K_{j} \text { for every } t \in I .
$$

Indeed, (2) is valid for $j=0$, since

$$
\left|\left(D_{q}^{(0)} h\right)(t)\right| \leqq K=K_{0} \text { for every } t \in I .
$$

We suppose that (2) is satisfied for $j=l, 0 \leqq l<m-1$, i.e.

$$
\left|\left(D_{q}^{(l)} h\right)(t)\right| \leqq K_{l} \text { for every } t \in I .
$$

For this $l$ we have that

$$
\left|\left(D_{q}^{(l+2)} h\right)(t)\right| \leqq S K^{1-(l+2) / m} M^{(l+2) / m} \equiv M_{l} \text { for every } t \in I
$$

which, by the definition of $S$, is obvious for $l<m-2$ and follows from the inequality

$$
\left|\left(D_{q}^{(m)} h\right)(t)\right| \leqq M \leqq M S=M_{m-2} \text { for every } t \in I,
$$

when $l=m-2$. Since, by (1),

$$
\left(\frac{K_{l}}{M_{l}}\right)^{1 / 2} \leqq\left(\frac{K}{M}\right)^{1 / m}
$$

and because of (a), there exist $t_{1}, t_{2}$ in $I$ with $t_{2}-t_{1}=2 A\left(K_{l} / M_{l}\right)^{1 / 2}$ and $t_{1} \leqq T_{0} \leqq t_{2}$, where $T_{0}$ is such that

$$
\left|\left(D_{q}^{(l+1)} h\right)\left(T_{0}\right)\right|=\max _{t \in I}\left|\left(D_{q}^{(l+1)} h\right)(t)\right| .
$$

It is easy to verify that

$$
\begin{aligned}
\left(D_{q}^{(l)} h\right)\left(t_{2}\right)-\left(D_{q}^{(l)} h\right)\left(T_{0}\right)= & \left(D_{q}^{(l+1)} h\right)\left(T_{0}\right) \int_{T_{0}}^{t_{2}} \frac{d s}{q_{l+1}(s)} \\
& +\int_{T_{0}}^{t_{2}} \frac{1}{q_{l+1}(s)} \int_{T_{0}}^{s} \frac{1}{q_{l+2}(w)}\left(D_{q}^{(l+2)} h\right)(w) d w d s
\end{aligned}
$$

and

$$
\begin{aligned}
\left(D_{q}^{(l)} h\right)\left(t_{1}\right)-\left(D_{q}^{(l)} h\right)\left(T_{0}\right)= & \left(D_{q}^{(l+1)} h\right)\left(T_{0}\right) \int_{T_{0}}^{t_{1}} \frac{d s}{q_{l+1}(s)} \\
& +\int_{T_{0}}^{t_{1}} \frac{1}{q_{l+1}(s)} \int_{T_{0}}^{s} \frac{1}{q_{l+2}(w)}\left(D_{q}^{(l+2)} h\right)(w) d w d s
\end{aligned}
$$


when, by subtraction, we get

$$
\begin{aligned}
\left(D_{q}^{(l)} h\right)\left(t_{2}\right)-\left(D_{q}^{(l)} h\right)\left(t_{1}\right)= & \left(D_{q}^{(l+1)} h\right)\left(T_{0}\right) \int_{t_{1}}^{t_{2}} \frac{d s}{q_{l+1}(s)} \\
& +\int_{T_{0}}^{t_{2}} \frac{1}{q_{l+1}(s)} \int_{T_{0}}^{s} \frac{1}{q_{l+2}(w)}\left(D_{q}^{(l+2)} h\right)(w) d w d s \\
& -\int_{l_{1}}^{T_{0}} \frac{1}{q_{l+1}(s)} \int_{s}^{T_{0}} \frac{1}{q_{l+2}(w)}\left(D_{q}^{(l+2)} h\right)(w) d w d s .
\end{aligned}
$$

Thus, we obtain

$$
\begin{aligned}
\left|\left(D_{q}^{(l+1)} h\right)\left(T_{0}\right)\right| \int_{t_{1}}^{t_{2}} \frac{d s}{q_{l+1}(s)} \leqq & \left|\left(D_{q}^{(l)} h\right)\left(t_{1}\right)\right|+\left|\left(D_{q}^{(l)} h\right)\left(t_{2}\right)\right| \\
& +\int_{t_{1}}^{T_{0}} \frac{1}{q_{l+1}(s)} \int_{s}^{T_{0}} \frac{1}{q_{l+2}(w)}\left|\left(D_{q}^{(l+2)} h\right)(w)\right| d w d s \\
& +\int_{T_{0}}^{t_{2}} \frac{1}{q_{l+1}(s)} \int_{T_{0}}^{s} \frac{1}{q_{l+2}(w)}\left|\left(D_{q}^{(l+2)} h\right)(w)\right| d w d s
\end{aligned}
$$

and consequently, by (3) and (4),

$$
\begin{gathered}
\left|\left(D_{q}^{(l+1)} h\right)\left(T_{0}\right)\right| \leqq \frac{1}{\int_{t_{1}}^{t_{2}} \frac{d s}{q_{l+1}(s)}}\left\{2 K_{l}+M_{l}\left[\int_{t_{1}}^{T_{0}} \frac{1}{q_{l+1}(s)} \int_{s}^{T_{0}} \frac{1}{q_{l+2}(w)} d w d s\right.\right. \\
\left.\left.+\int_{T_{0}}^{t_{2}} \frac{1}{q_{l+1}(s)} \int_{T_{0}}^{s} \frac{1}{q_{l+2}(w)} d w d s\right]\right\} .
\end{gathered}
$$

Hence,

$$
\begin{aligned}
\left|\left(D_{q}^{(l+1)} h\right)\left(T_{0}\right)\right| & \leqq \frac{B}{t_{2}-t_{1}}\left\{2 K_{l}+\frac{M_{l}}{2 A^{2}}\left[\left(T_{0}-t_{1}\right)^{2}+\left(t_{2}-T_{0}\right)^{2}\right]\right\} \\
& \leqq \frac{B}{t_{2}-t_{1}}\left[2 K_{l}+\frac{M_{l}}{2 A^{2}}\left(t_{2}-t_{1}\right)^{2}\right] \\
& =B\left[\frac{2 K_{l}}{t_{2}-t_{1}}+\frac{M_{l}}{2 A^{2}}\left(t_{2}-t_{1}\right)\right] \\
& =\frac{2 B}{A}\left(K_{l} M_{l}\right)^{1 / 2}
\end{aligned}
$$

But, it is a matter of calculations to verify that

$$
\frac{2 B}{A}\left(K_{l} M_{l}\right)^{1 / 2}=K_{l+1}
$$


when

$$
\left(D_{q}^{(l+1)} h\right)\left(T_{0}\right) \mid \leqq K_{l+1},
$$

which proves (2) for $j=l+1$ and consequently for all $j=0,1, \cdots, m-1$.

From (2) it follows that

$$
\max _{0<j<m} \max _{t \in I} \frac{\left|\left(D_{q}^{(j)} h\right)(t)\right|}{K^{1-j / m} M^{j / m}} \leqq\left(\frac{4 B^{2}}{A^{2}} S\right)^{\gamma_{m-1}}
$$

Also,

$$
c_{m}=\left(\frac{2 B}{A}\right)^{2^{m-2}}=\left(\frac{4 B^{2}}{A^{2}} c_{m}\right)^{\gamma_{m-1}} \leqq\left(\frac{4 B^{2}}{A^{2}} S\right)^{\gamma_{m-1}} .
$$

Therefore, by the definition of $S$,

$$
S \leqq\left(\frac{4 B^{2}}{A^{2}} S\right)^{\gamma_{m-1}}
$$

which gives

$$
S \leqq\left(\frac{2 B}{A}\right)^{2^{m-2}}=c_{m}, \quad \text { i.e. } \quad S=c_{m} .
$$

Hence, by the definition of $S$, we have that for every $t \in I$

$$
\left|\left(D_{q}^{(j)} h\right)(t)\right| \leqq c_{m} K^{1-j / m} M^{j / m} \quad(j=1,2, \cdots, m-1) .
$$

Finally, for our purpose we need one more lemma.

LEMMA 3. Let $q_{i}(i=0,1, \cdots, \lambda)$ be positive continuous functions on an interval $[T, \infty)$ such that for $\lambda>0$,

$$
\int^{\infty} \frac{d t}{q_{i}(t)}=\infty \quad(i=1,2, \cdots, \lambda)
$$

and let

$$
Q_{0 \lambda}(t ; T)=\left\{\begin{array}{l}
1, \quad \text { if } \lambda=0 \\
\int_{T}^{t} \frac{1}{q_{1}\left(s_{1}\right)} \int_{T}^{s_{1}} \frac{1}{q_{2}\left(s_{2}\right)} \cdots \int_{T}^{s_{\lambda-1}} \frac{1}{q_{\lambda}\left(s_{\lambda}\right)} d s_{\lambda} \cdots d s_{2} d s_{1}, \\
\text { if } \lambda>0 .
\end{array}\right.
$$


Moreover, let $h$ be a $\lambda$-times continuously $q$-differentiable function on the interval $[T, \infty)$. Then we have:

( $\alpha)$ If

$$
\liminf _{t \rightarrow \infty}\left(D_{q}^{(\lambda)} h\right)(t)>0 \quad \text { or } \quad \limsup _{t \rightarrow \infty}\left(D_{q}^{(\lambda)} h\right)(t)<0
$$

and

$$
\int^{\infty} \frac{Q_{0 \lambda}(t ; T)}{q_{0}(t)} d t=\infty
$$

then

$$
\int^{\infty} h(t) d t= \pm \infty
$$

( $\beta$ ) If

$$
\lim _{t \rightarrow \infty}\left(D_{q}^{(\lambda)} h\right)(t)=0
$$

and

$$
\limsup _{t \rightarrow \infty} \frac{Q_{0 \lambda}(t ; T)}{q_{0}(t)}<\infty
$$

then

$$
\lim _{t \rightarrow \infty} h(t)=0
$$

Proof. Since the lemma is obvious for $\lambda=0$, we suppose that $\lambda>0$. For any integers $i$ and $j, 0 \leqq i \leqq j \leqq \lambda$, and for every $u$ and $v$ with $v \geqq u \geqq T$, we define

$$
Q_{i j}(v ; u)=\left\{\begin{array}{l}
1, \text { if } i=j \\
\int_{u}^{v} \frac{1}{q_{t+1}\left(s_{t+1}\right)} \int_{u}^{s_{t+1}} \frac{1}{q_{i+2}\left(s_{t+2}\right)} \cdots \int_{u}^{s_{i-1}} \frac{1}{q_{\jmath}\left(s_{\jmath}\right)} d s_{j} \cdots d s_{i+2} d s_{i+1},
\end{array}\right.
$$


Then, taking into account condition (I), we have that for every $T^{*} \geqq T$,

$$
\lim _{t \rightarrow \infty} Q_{i j}\left(t ; T^{*}\right)=\infty \quad(0 \leqq i<j \leqq \lambda)
$$

and consequently, by L'Hospital's rule,

$$
\lim _{t \rightarrow \infty} \frac{Q_{0 j}\left(t ; T^{*}\right)}{Q_{0 \lambda}\left(t ; T^{*}\right)}=0 \quad(j=0,1, \cdots, \lambda-1)
$$

and

$$
\lim _{t \rightarrow \infty} \frac{Q_{0 \lambda}\left(t ; T^{*}\right)}{Q_{0 \lambda}(t ; T)}=1
$$

Moreover, for every $t$ and $T^{*}$ with $t \geqq T^{*} \geqq T$, it is easy to derive the following generalized Taylor's formula

$$
\begin{aligned}
\left(D_{q}^{(0)} h\right)(t) & =\sum_{j=0}^{\lambda-1}\left(D_{q}^{(j)} h\right)\left(T^{*}\right) Q_{0 j}\left(t ; T^{*}\right) \\
& (7) \quad+\int_{T^{*}}^{t} \frac{1}{q_{1}\left(s_{1}\right)} \int_{T^{*}}^{s_{1}} \frac{1}{q_{2}\left(s_{2}\right)} \cdots \int_{T^{*}}^{s_{\lambda}-1} \frac{1}{q_{\lambda}\left(s_{\lambda}\right)}\left(D_{q}^{(\lambda)} h\right)\left(s_{\lambda}\right) d s_{\lambda} \cdots d s_{2} d s_{1_{1}}
\end{aligned}
$$

( $\alpha$ ) Let $d>0$ and $T^{*}>T$ be chosen so that

$$
\left(D_{q}^{(\lambda)} h\right)(t) \geqq d \text { for every } t \geqq T^{*}
$$

or

$$
\left(D_{q}^{(\lambda)} h\right)(t) \leqq-d \text { for every } t \geqq T^{*} \text {. }
$$

Then from the formula (7) we have respectively

$$
\frac{\left(D_{q}^{(0)} h\right)(t)}{Q_{o \lambda}(t ; T)} \geqq \sum_{j=0}^{\lambda-1}\left(D_{q}^{(j)} h\right)\left(T^{*}\right) \frac{Q_{0 j}\left(t ; T^{*}\right)}{Q_{0 \lambda}(t ; T)}+d \frac{Q_{0 \lambda}\left(t ; T^{*}\right)}{Q_{0 \lambda}(t ; T)}, \quad t \geqq T^{*}
$$

or

$$
\frac{\left(D_{q}^{(0)} h\right)(t)}{Q_{0 \lambda}(t ; T)} \leqq \sum_{j=0}^{\lambda-1}\left(D_{q}^{(j)} h\right)\left(T^{*}\right) \frac{Q_{0 j}\left(t ; T^{*}\right)}{Q_{0 \lambda}(t ; T)}-d \frac{Q_{0 \lambda}\left(t ; T^{*}\right)}{Q_{0 \lambda}(t ; T)}, \quad t \geqq T^{*}
$$

and hence, by virtue of (5) and (6), we obtain

$$
\liminf _{t \rightarrow \infty} \frac{\left(D_{q}^{(0)} h\right)(t)}{Q_{0 \lambda}(t ; T)}>0 \quad \text { or } \quad \limsup _{t \rightarrow \infty} \frac{\left(D_{q}^{(0)} h\right)(t)}{Q_{0 \lambda}(t ; T)}<0
$$


But this, by condition (II), gives

$$
\int^{\infty} h(t) d t= \pm \infty
$$

( $\beta$ ) For any arbitrary $\epsilon>0$, we consider a $T^{*}>T$ so that

$$
\left|\left(D_{q}^{(\lambda)} h\right)(t)\right|<\epsilon \text { for every } t \geqq T^{*},
$$

when from the formula (7) we obtain

$$
\frac{\left\lfloor\left(D_{q}^{(0)} h\right)(t)\right\rfloor}{Q_{0 \lambda}(t ; T)} \leqq \sum_{j=0}^{\lambda-1}\left|\left(D_{q}^{(1)} h\right)\left(T^{*}\right)\right| \frac{Q_{0 j}\left(t ; T^{*}\right)}{Q_{0 \lambda}(t ; T)}+\epsilon \frac{Q_{0 \lambda}\left(t ; T^{*}\right)}{Q_{0 \lambda}(t ; T)}
$$

for every $t \geqq T^{*}$. Thus, by (5) and (6), we have

$$
\limsup _{t \rightarrow \infty} \frac{\left\lfloor\left(D_{q}^{(0)} h\right)(t) \mid\right.}{Q_{0 \lambda}(t ; T)} \leqq \epsilon
$$

and, since $\epsilon$ is arbitrary,

$$
\lim _{t \rightarrow \infty} \frac{\left(D_{q}^{(0)} h\right)(t)}{Q_{0 \lambda}(t ; T)}=0
$$

which, by condition (III), gives

$$
\lim _{t \rightarrow \infty} h(t)=0
$$

2. The main result. Our main theorem establishes conditions which essentially guarantee that

$$
\lim _{t \rightarrow \infty} x(t)=0
$$

for the bounded nonoscillatory solutions $x$ of the equation (E).

THEOREM. Consider the differential equation (E) subject to the conditions (i), (ii) and

(iii) $\lim \sup _{t \rightarrow \infty} r_{0}(t)<\infty$.

Let $m$ and $k$ be integers with $1 \leqq m \leqq k \leqq n-1$ so that the conditions (iv), (v) and (vi) below are satisfied:

(iv) If $m<k$, then for every $j=m+1, \cdots, k$

$$
\int^{\infty} \frac{d t}{r_{l}(t)}=\infty
$$


(v) $\int^{\infty} \frac{R_{m k}(t)}{r_{m}(t)} d t=\infty$ and $\quad \limsup _{t \rightarrow \infty} \frac{R_{m k}(t)}{r_{m}(t)}<\infty$,

where

$$
R_{m k}(t)=\left\{\begin{array}{l}
1, \text { if } m=k \\
\int_{t_{0}}^{t} \frac{1}{r_{m+1}\left(s_{m+1}\right)} \int_{t_{0}}^{s_{m+1}} \frac{1}{r_{m+2}\left(s_{m+2}\right)} \cdots \int_{t_{0}}^{s_{k-1}} \frac{1}{r_{k}\left(s_{k}\right)} d s_{k} \cdots d s_{m+2} d s_{m+1}, \\
\text { if } \quad m<k .
\end{array}\right.
$$

(vi) If $m>1$, then for every $i=1,2, \cdots, m-1$

$$
0<\liminf _{t \rightarrow \infty} r_{i}(t) \leqq \limsup _{t \rightarrow \infty} r_{i}(t)<\infty
$$

Moreover, let there exist a function $\rho$ of the type $r[k]$ such that:

$$
\int^{\infty} \rho(t)|b(t)| d t<\infty
$$

and

$\left(\mathrm{C}_{2}\right)$ For some $\delta>0$,

$$
\liminf _{t \rightarrow \infty} \int_{t}^{t+\delta} \rho(s) a^{+}(s) d s>0 \text { and } \int^{\infty} \rho(t) a^{-}(t) d t<\infty
$$

or

$$
\liminf _{t \rightarrow \infty} \int_{t}^{t+\delta} \rho(s) a^{-}(s) d s>0 \text { and } \int^{\infty} \rho(t) a^{+}(t) d t<\infty
$$

where

$$
a^{+}(t)=\max \{a(t), 0\} \quad \text { and } \quad a^{-}(t)=\max \{-a(t), 0\} .
$$

If the function $\sigma$ is differentiable with bounded derivative on $\left[t_{0}, \infty\right)$, then for all bounded nonoscillatory solutions $x$ of the equation (E),

$$
\lim _{t \rightarrow \infty}\left(D_{r}^{(k)} x\right)(t)=0=\lim _{t \rightarrow \infty}\left(D_{r}^{(m-1)} x\right)^{\prime}(t)
$$




$$
\lim _{t \rightarrow \infty}\left(D_{r}^{(l)} x\right)(t)=0 \quad(l=0,1, \cdots, m-1)
$$

Proof. Let $x$ be a bounded nonoscillatory solution of (E). Without loss of generality, we assume that $x$ is a solution on the whole interval $\left[t_{0}, \infty\right)$. Moreover, this solution is supposed positive on $\left[t_{0}, \infty\right)$, since the substitution $u=-x$ transforms (E) into an equation of the same form satisfying the assumptions of the theorem.

If, by (i), $T>t_{0}$ is chosen so that for every $t \geqq T$

$$
\sigma(t) \geqq t_{0}
$$

then from equation $(E)$ we obtain

$$
\int_{T}^{t} \rho(s)\left(D_{r}^{(n)} x\right)(s) d s=\int_{T}^{t} \rho(s) b(s) d s-\int_{T}^{t} \rho(s) a^{+}(s) F(x[\sigma(s)]) d s
$$

$$
+\int_{T}^{t} \rho(s) a^{-}(s) F(x[\sigma(s)]) d s
$$

for every $t \geqq T$. Because of (ii) and the boundedness of $x$, the function $F \circ x \circ \sigma$ is positive and bounded on $[T, \infty)$. Thus, by conditions $\left(\mathrm{C}_{1}\right)$ and $\left(C_{2}\right)$, we conclude from (8) that the improper integral

$$
\int_{T}^{\infty} \rho(t)\left(D_{r}^{(n)} x\right)(t) d t
$$

exists in $\mathbf{R}^{*}$ and consequently, by virtue of Lemma 1 , the $\lim _{t \rightarrow \infty}\left(D_{r}^{(k)} x\right)(t)$ also exists in $\mathbf{R}^{*}$. Moreover,

$$
\lim _{t \rightarrow \infty}\left(D_{r}^{(k)} x\right)(t)=0
$$

Indeed, in the opposite case we have

$$
\liminf _{t \rightarrow \infty}\left(D_{r}^{(k)} x\right)(t)>0 \quad \text { or } \quad \limsup _{t \rightarrow \infty}\left(D_{r}^{(k)} x\right)(t)<0 .
$$

Thus, because of conditions (iv) and (v), we can apply Lemma 3(a) for $h=\left(D_{r}^{(m-1)} x\right)^{\prime}, \lambda=k-m$ and $q_{j}=r_{m+j}(j=0,1, \cdots, \lambda)$, when we obtain

$$
\int^{\infty}\left(D_{r}^{(m-1)} x\right)^{\prime}(t) d t= \pm \infty, \quad \text { i.e. } \quad \lim _{t \rightarrow \infty}\left(D_{r}^{(m-1)} x\right)(t)= \pm \infty \text {. }
$$

From this, by condition (vi), it is easy to derive 


$$
\lim _{t \rightarrow \infty}\left(D_{r}^{(0)} x\right)(t)= \pm \infty
$$

a contradiction, since, by condition (iii), $D_{r}^{(0)} x=r_{0} x$ is bounded.

Now, taking into account (9) and conditions (iv) and (v), we apply again Lemma $3(\beta)$ for $h=\left(D_{r}^{(m-1)} x\right)^{\prime}, \lambda=k-m$ and $q_{j}=r_{m+j}$ $(j=0,1, \cdots, \lambda)$, when we get

$$
\lim _{t \rightarrow \infty}\left(D_{r}^{(m-1)} x\right)^{\prime}(t)=0 .
$$

So, if $m>1$, then, by virtue of condition (vi), we can apply Lemma 2 for $q_{j}=r_{j}(j=0,1, \cdots, m-1)$ and $q_{m}=1$, to obtain

$$
\lim _{t \rightarrow \infty}\left(D_{r}^{(j)} x\right)(t)=0 \quad(j=1,2, \cdots, m-1) .
$$

Thus, it remains to prove that

$$
\lim _{t \rightarrow \infty}\left(D_{r}^{(0)} x\right)(t)=0 .
$$

To do this, we first observe that

$$
\liminf _{t \rightarrow \infty}\left(D_{r}^{(0)} x\right)(t)=0
$$

Indeed, in the opposite case for some positive constant $d_{1}$ and for every $t \geqq t_{0}$ we have

$$
\left(D_{r}^{(0)} x\right)(t) \geqq d_{1}
$$

and consequently, by (iii),

$$
x(t) \geqq d_{2} \text { for every } t \geqq t_{0}
$$

where $d_{2}=d_{1} / \sup _{t \geqq \iota_{1}} r_{0}(t)$. From this and the boundedness of $x$ we conclude that

$$
F(x[\sigma(t)]) \geqq d \text { for every } t \geqq T,
$$

where $d$ is a positive constant. Hence, from (8) and on account of conditions $\left(C_{1}\right)$ and $\left(C_{2}\right)$, it follows that

$$
\int_{T}^{\infty} \rho(t)\left(D_{r}^{(n)} x\right)(t) d t= \pm \infty
$$


and consequently, by applying Lemma 1,

$$
\lim _{t \rightarrow \infty}\left(D_{r}^{(k)} x\right)(t)= \pm \infty
$$

which contradicts (9).

To complete the proof of (12), we have verify that

$$
\limsup _{t \rightarrow \infty}\left(D_{r}^{(0)} x\right)(t)=0
$$

Indeed, in the opposite case we have

$$
\limsup _{t \rightarrow \infty}\left(D_{r}^{(0)} x\right)[\sigma(t)] \geqq K
$$

for some positive constant $K$. Hence, on account of (13) and based on the arguments of Hammett [3] (cf. also Singh [7,8] and Staikos and Philos [9]), we derive that there exist three sequences $\left(\alpha_{\nu}\right),\left(\beta_{\nu}\right)$ and $\left(\gamma_{\nu}\right)$ with $\lim \alpha_{\nu}=\infty$ and such that for every $\nu=1,2, \cdots$

$$
\begin{gathered}
T \leqq \alpha_{\nu}<\gamma_{\nu}<\beta_{\nu} \leqq \alpha_{\nu+1} \\
\left(D_{r}^{(0)} x\right)\left[\sigma\left(\alpha_{\nu}\right)\right]=\frac{K}{2}=\left(D_{r}^{(0)} x\right)\left[\sigma\left(\beta_{\nu}\right)\right] \\
\left(D_{r}^{(0)} x\right)\left[\sigma\left(\gamma_{\nu}\right)\right]>K \\
\left(D_{r}^{(0)} x\right)[\sigma(t)]>\frac{K}{2} \text { for every } t \in\left(\alpha_{\nu}, \beta_{\nu}\right) .
\end{gathered}
$$

By mean-value theorem, we have

$$
\frac{\left(D_{r}^{(0)} x\right)\left[\sigma\left(\gamma_{\nu}\right)\right]-\left(D_{r}^{(0)} x\right)\left[\sigma\left(\alpha_{\nu}\right)\right]}{\gamma_{\nu}-\alpha_{\nu}}=\sigma^{\prime}\left(\xi_{\nu}\right)\left(D_{r}^{(0)} x\right)^{\prime}\left[\sigma\left(\xi_{\nu}\right)\right]
$$

and consequently

$$
\frac{K}{2\left(\beta_{\nu}-\alpha_{\nu}\right)}<\sigma^{\prime}\left(\xi_{\nu}\right)\left(D_{r}^{(0)} x\right)^{\prime}\left[\sigma\left(\xi_{\nu}\right)\right]
$$

where obviously $\lim \xi_{\nu}=\infty$. But, because of (10), (11) and (vi), it is easy to see that

$$
\lim _{t \rightarrow \infty}\left(D_{r}^{(0)} x\right)^{\prime}(t)=0
$$


Thus, we obtain

$$
\lim \left(\beta_{\nu}-\alpha_{\nu}\right)=\infty
$$

Next, we observe that for every $\nu=1,2, \cdots$

$$
\left(D_{r}^{(0)} x\right)[\sigma(t)] \geqq \frac{K}{2} \text { for every } t \in\left[\alpha_{\nu}, \beta_{\nu}\right]
$$

and consequently, by (iii),

$$
x[\sigma(t)] \geqq \frac{K}{2 r_{0}[\sigma(t)]} \geqq \frac{K}{2 \sup _{t \geqq \iota} r_{0}(t)}>0 \text { for every } t \in\left[\alpha_{\nu}, \beta_{\nu}\right]
$$

We have thus proved that the bounded function $x \circ \sigma$ has a positive lower bound on the set $\bigcup_{\nu=1}^{\infty}\left[\alpha_{\nu}, \beta_{\nu}\right]$. Hence, because of (ii), we have

$$
F(x[\sigma(t)]) \geqq M \text { for every } t \in \bigcup_{\nu=1}^{\infty}\left[\alpha_{\nu}, \beta_{\nu}\right]
$$

where the constant $M$ is positive.

Obviously,

$$
\begin{aligned}
\int_{T}^{\infty} \rho(t) a^{ \pm}(t) F(x[\sigma(t)]) d t & \geqq \sum_{\nu=1}^{\infty} \int_{\alpha_{\nu}}^{\beta_{\nu}} \rho(t) a^{ \pm}(t) F(x[\sigma(t)]) d t \\
& \geqq M \sum_{\nu=1}^{\infty} \int_{\alpha_{\nu}}^{\beta_{\nu}} \rho(t) a^{ \pm}(t) d t
\end{aligned}
$$

But, by virtue of (14) and condition $\left(\mathrm{C}_{2}\right)$, we have

$$
\sum_{\nu=1}^{\infty} \int_{\alpha_{\nu}}^{\beta_{\nu}} \rho(t) a^{+}(t) d t=\infty \quad \text { or } \quad \sum_{\nu=1}^{\infty} \int_{\alpha_{\nu}}^{\beta_{\nu}} \rho(t) a^{-}(t) d t=\infty
$$

and consequently

$$
\int_{T}^{\infty} \rho(t) a^{+}(t) F(x[\sigma(t)]) d t=\infty \quad \text { or } \quad \int_{T}^{\infty} \rho(t) a^{-}(t) F(x[\sigma(t)]) d t=\infty
$$

Thus, from (8) it follows that

$$
\int_{T}^{\infty} \rho(t)\left(D_{r}^{(n)} x\right)(t) d t= \pm \infty
$$


Finally, by Lemma 1 , we obtain

$$
\lim _{t \rightarrow \infty}\left(D_{r}^{(k)} x\right)(t)= \pm \infty
$$

which contradicts (9).

3. Applications. We shall give now some interesting applications of our main result for the particular case

$$
r_{j}=1 \text { for } j \neq n-N \text { and } r_{n-N}=r \text {, }
$$

where $N$ is an integer with $1 \leqq N \leqq n-1$. More precisely, we shall derive some corollaries concerning the differential equation

$\left(\mathrm{D}_{N}\right) \quad\left[r(t) x^{(n-N)}(t)\right]^{(N)}+a(t) F(x[\sigma(t)])=b(t), \quad t \geqq t_{0}$.

All corollaries are new except Corollary 1, which is the main result of a recent paper by Kusano and Onose [5].

COROllary 1. Consider the differential equation $\left(\mathrm{D}_{N}\right)$ subject to the conditions (i) and (ii). Moreover, let $k, 0 \leqq k \leqq N-1$, be an integer such that:

( $\alpha) \quad \int^{\infty} \frac{t^{N-1-k}}{r(t)} d t=\infty$ and $\quad \limsup _{t \rightarrow \infty} \frac{t^{N-1-k}}{r(t)}<\infty$,

$$
\int^{\infty} t^{k}|b(t)| d t<\infty
$$

and

$(\gamma)$ For some $\delta>0$,

$$
\liminf _{t \rightarrow \infty} \int_{t}^{t+\delta} s^{k} a^{+}(s) d s>0 \text { and } \int^{\infty} t^{k} a^{-}(t) d t<\infty
$$

or

$$
\liminf _{t \rightarrow \infty} \int_{t}^{t+\delta} s^{k} a^{-}(s) d s>0 \text { and } \int^{\infty} t^{k} a^{+}(t) d t<\infty
$$

If the function $\sigma$ is differentiable with bounded derivative on $\left[t_{0}, \infty\right)$, then for all bounded nonoscillatory solutions $x$ of the differential equation $\left(\mathrm{D}_{N}\right)$, 


$$
\lim _{t \rightarrow \infty}\left[r(t) x^{(n-N)}(t)\right]^{(N-1-k)}=0
$$

and

$$
\lim _{t \rightarrow \infty} x^{(i)}(t)=0 \quad(i=0,1, \cdots, n-N)
$$

Proof. It is easy to see that the function $\rho$,

$$
\rho(t)=\left(t-t_{0}\right)^{k}
$$

is of the type $r[n-1-k]$. Thus, the corollary follows by applying our theorem for $m=n-N$ and with $n-1-k$ in place of $k$.

Corollary 2. Consider the differential equation $\left(\mathrm{D}_{N}\right)$ with $N>1$, subject to the conditions (i), (ii) and

$$
0<\liminf _{t \rightarrow \infty} r(t) \leqq \limsup _{t \rightarrow \infty} r(t)<\infty
$$

Moreover, let $k, 0 \leqq k<N-1$, be an integer such that $(\beta)$ and $(\gamma)$ are satisfied.

If the function $\sigma$ is differentiable with bounded derivative on $\left[t_{0}, \infty\right)$, then for all bounded nonoscillatory solutions $x$ of the differential equation $\left(\mathrm{D}_{N}\right)$,

$$
\lim _{t \rightarrow \infty}\left[r(t) x^{(n-N)}(t)\right]^{(j)}=0 \quad(j=0,1, \cdots, N-1-k)
$$

and

$$
\lim _{i \rightarrow \infty} x^{(i)}(t)=0 \quad(i=0,1, \cdots, n-N)
$$

Proof. It follows from our theorem, by taking the same function $\rho$ as in the proof of Corollary $1, m=n-1-k>n-N$ and $n-1-k$ in place of $k$.

Corollary 3. Consider the differential equation $\left(\mathrm{D}_{N}\right)$ with $N<$ $n-1$, subject to the conditions (i) and (ii). Moreover, let $k, 1 \leqq k \leqq$ $n-N-1$, be an integer such that:

$$
\int \frac{t^{n-N-1-k}}{r(t)} d t=\infty
$$


$(\epsilon)$

$$
\int^{\infty} \rho_{k}(t)|b(t)| d t<\infty
$$

and for some $\delta>0$ either

$$
\liminf _{t \rightarrow \infty} \int_{t}^{t+\delta} \rho_{k}(s) a^{+}(s) d s>0 \quad \text { and } \quad \int^{\infty} \rho_{k}(t) a^{-}(t) d t<\infty
$$

or

$$
\liminf _{t \rightarrow x} \int_{t}^{t+\delta} \rho_{k}(s) a^{-}(s) d s>0 \quad \text { and } \quad \int^{\infty} \rho_{k}(t) a^{+}(t) d t<\infty
$$

where

$$
\rho_{k}(t)=\int_{t_{0}}^{t} \frac{(t-s)^{N-1}\left(s-t_{0}\right)^{n-N-1-k}}{r(s)} d s
$$

If the function $\sigma$ is differentiable with bounded derivative on $\left[t_{0}, \infty\right)$, then for all bounded nonoscillatory solutions $x$ of the differential equation $\left(\mathrm{D}_{N}\right)$,

$$
\lim _{t \rightarrow \infty} x^{(t)}(t)=0 \quad(i=0,1, \cdots, k)
$$

Proof. Here, we have to apply our theorem for $m=k$ and $\rho=\rho_{k}$, since, as it is easy to see, the function $\rho_{k}$ is of the type $r[k]$.

COROllary 4. Consider the differential equation $\left(\mathrm{D}_{N}\right)$ with $N<$ $n-1$, subject to the conditions (i) and (ii). Moreover, let $k, 1 \leqq k \leqq$ $n-N-1$ be an integer such that:

$$
\int^{\infty} \frac{t^{n-N-1-k}}{r(t)} d t<\infty
$$

( $\eta$ If $N>1$, then for every $j=1,2, \cdots, N-1$

$$
\begin{gathered}
\lim _{t \rightarrow \infty} \int_{t^{\prime}}^{t}(t-s)^{\prime-1} \int_{s}^{\infty} \frac{\left(u-t_{0}\right)^{n-N-1-k}}{r(u)} d u d s \text { exists in }\{0, \infty\} \\
\int^{\infty} \rho_{k}(t)|b(t)| d t<\infty
\end{gathered}
$$

and for some $\delta>0$ either

$$
\liminf _{t \rightarrow \infty} \int_{t}^{t+\delta} \rho_{k}(s) a^{+}(s) d s>0 \text { and } \quad \int^{\infty} \rho_{k}(t) a^{-}(t) d t<\infty
$$


or

$$
\liminf _{t \rightarrow \infty} \int_{t}^{t+\delta} \rho_{k}(s) a^{-}(s) d s>0 \text { and } \int^{\infty} \rho_{k}(t) a^{+}(t) d t<\infty,
$$

where

$$
\rho_{k}(t)=\left\{\begin{array}{l}
\int_{t}^{\infty} \frac{\left(s-t_{0}\right)^{n-2-k}}{r(s)} d s, \text { if } N=1 \\
\int_{t_{0}}^{1}(t-s)^{N-2} \int_{s}^{\infty} \frac{\left(u-t_{0}\right)^{n-N-1-k}}{r(u)} d u d s, \quad \text { if } \quad N>1 .
\end{array}\right.
$$

If the function $\sigma$ is differentiable with bounded derivative on $\left[t_{0}, \infty\right)$, then for all bounded nonoscillatory solutions $x$ of the differential equation $\left(\mathrm{D}_{N}\right)$,

$$
\lim _{t \rightarrow \infty} x^{(i)}(t)=0 \quad(i=0,1, \cdots, k)
$$

Proof. It is easy to verify that the function $\rho_{k}$ is of the type $r[k]$, when the corollary follows immediately by applying our theorem for $m=k$.

The significance of Corollaries 2, 3 and 4 is illustrated by the three examples below, where in each case exactly one of these corollaries can be applied and in addition Corollary 1 fails.

EXAMPLE 1. The retarded differential equation

$$
\left[\left(1+e^{-t}\right) x^{\prime}(t)\right]^{\prime \prime}+\frac{1}{t} x^{2}(\log t) \operatorname{sgn} x(\log t)=\frac{1}{t^{3}}+e^{-t}\left(1+8 e^{-t}\right), \quad t \geqq 1
$$

has the bounded nonoscillatory solution $x(t)=e^{-t}$ with the property

$$
\lim _{t \rightarrow \infty} x(t)=\lim _{t \rightarrow \infty} x^{\prime}(t)=\lim _{t \rightarrow \infty}\left[\left(1+e^{-t}\right) x^{\prime}(t)\right]^{\prime}=0
$$

Further, as it follows from Corollary 2, every bounded nonoscillatory solution $x$ of the above equation has this property.

Example 2. By Corollary 3, all bounded nonoscillatory solutions of the differential equation

$$
\left[t^{1 / 2} x^{\prime \prime}(t)\right]^{\prime}+t^{-1 / 2} x^{5}(\gamma t)=\left(\gamma^{-5 / 2}-3 / 2\right) t^{-3}, \quad t \geqq 1,
$$


where $\gamma$ is a positive constant, tend to zero as $t \rightarrow \infty$ together with their derivatives. For example, $x(t)=t^{-1 / 2}$ is a bounded nonoscillatory solution of the above equation with $\lim _{t \rightarrow \infty} x(t)=\lim _{t \rightarrow \infty} x^{\prime}(t)=0$.

EXAmPle 3. Consider the retarded differential equation

$$
\left[t^{2} x^{\prime \prime}(t)\right]^{\prime}+t x^{6}(\sqrt{t}) \operatorname{sgn} x(\sqrt{t})=-\frac{1}{t^{2}}, \quad t \geqq 1
$$

This equation has the bounded nonoscillatory solution $x(t)=1 / t$ with $\lim _{t \rightarrow \infty} x(t)=\lim _{t \rightarrow \infty} x^{\prime}(t)=0$. Further, from Corollary 4 it follows that all bounded nonoscillatory solutions of the considered equation tend to zero as $t \rightarrow \infty$ together with their derivatives.

REMARK. The results of this paper can be formulated in the case of more general differential equations of the form

$$
\left(D_{r}^{(n)} x\right)(t)+a(t) F(x[\sigma(t)])
$$$$
+G\left(t ; x\left\langle\tau_{0}(t)\right\rangle ;\left(D_{r}^{(1)} x\right)\left\langle\tau_{1}(t)\right\rangle, \cdots,\left(D_{r}^{(n-1)} x\right)\left\langle\tau_{n-1}(t)\right\rangle\right)=b(t), \quad t \geqq t_{0}
$$

where

$$
g\left\langle\tau_{i}(t)\right\rangle=\left(g\left[\tau_{11}(t)\right], g\left[\tau_{i 2}(t)\right], \cdots, g\left[\tau_{i \mu_{i}}(t)\right]\right)
$$

From the proof of our theorem, this is obvious under additional conditions on $G$, which ensure that for every bounded nonoscillatory and $n$-times continuously $r$-differentiable function $u$ on an interval $[T, \infty)$,

$$
\int^{\infty} \rho(t)\left|G\left(t ; u\left\langle\tau_{0}(t)\right\rangle ;\left(D_{r}^{(1)} u\right)\left\langle\tau_{1}(t)\right\rangle, \cdots,\left(D_{r}^{(n-1)} u\right)\left\langle\tau_{n-1}(t)\right\rangle\right)\right| d t<\infty,
$$

where $\rho$ is the function introduced in conditions $\left(C_{1}\right)$ and $\left(C_{2}\right)$ of our theorem. For a such related result to Corollary 1 see Chen [1].

\section{REFERENCES}

1. Lu-San Chen, On the nonoscillatory properties of solutions of a functional differential equation, Bull. Soc. Math. Grèce, to appear.

2. W. A. Coppel, Stability and Asymptotic Behavior of Differential Equations, D. C. Heath, Boston, 1965.

3. M. E. Hammett, Nonoscillation properties of a nonlinear differential equation, Proc. Amer. Math. Soc., 30 (1971), 92-96. 
4. G. H. Hardy and J. E. Littlewood, Contributions to the arithmetic theory of series, Proc. London Math. Soc., 11 (1913), 411-478.

5. T. Kusano and H. Onose, Asymptotic behavior of nonoscillatory solutions of nonlinear differential equations with forcing term, Ann. Mat. Pura Appl., 112 (1977), 231-240.

6. E. Landau, Über einen Satz von Herrn Esclangon, Math. Ann., 102 (1930), 177-188.

7. B. Singh, Nonoscillation of forced fourth order retarded equations, SIAM J. Appl. Math., 28 (1975), 265-269.

8. - Asymptotic nature of nonoscillatory solutions of nth order retarded differential equations, SIAM J. Math. Anal., 6 (1975), 784-795.

9. V. A. Staikos and Ch. G. Philos, On the asymptotic behavior of nonoscillatory solutions of differential equations with deviating arguments, Hiroshima Math. J., 7 (1977), 9-31.

Received March 16, 1976 and in revised form January 19, 1977.

UNIVERSITY OF IOANNINA

IOANNINA, GREECE 



\section{Pacific Journal of Mathematics \\ Vol. 70, No. $1 \quad$ September, 1977}

William H. Barker, Noether's theorem for plane domains with hyperelliptic

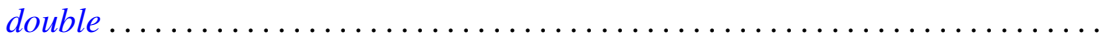

Michael James Beeson, Non-continuous dependence of surfaces of least area on the boundary curve ...................................... 11

Horst Behncke, Functions acting in weighted Orlicz algebras . . . . . . . . . . . . 19

Howard Edwin Bell, A commutativity study for periodic rings . . . . . . . . . . . 29

Peter Botta and Stephen J. Pierce, The preservers of any orthogonal group ....... 37

Douglas S. Bridges, The constructive Radon-Nikodým theorem ............. 51

James Dennis Brom, The theory of almost periodic functions in constructive

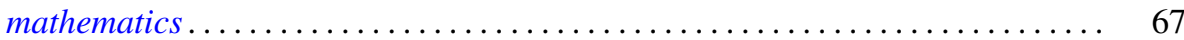

N. Burgoyne and C. Williamson, Semi-simple classes in Chevalley type groups ....

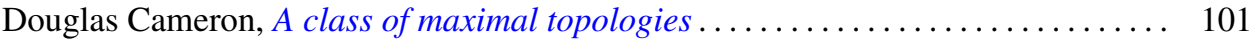

L. Carlitz, Enumeration of doubly up-down permutations . . . . . . . . . . . . . . 105

Paul Robert Chernoff, The quantum n-body problem and a theorem of

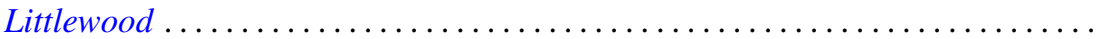

Jo-Ann Deborah Cohen, Locally bounded topologies on $F(X) \ldots \ldots \ldots \ldots \ldots \ldots$

Heinz Otto Cordes and Robert Colman McOwen, Remarks on singular elliptic

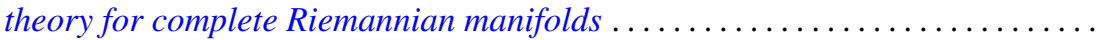

Micheal Neal Dyer, Correction to: "Rational homology and Whitehead

products"

Robert Fernholz, Factorization of Radonifying transformations

Lawrence Arthur Fialkow, A note on quasisimilarity. II ...... . .

Harvey Charles Greenwald, Lipschitz spaces of distributions on the surface of unit

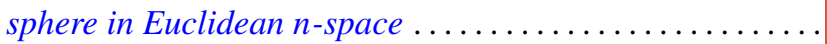

Albrecht Irle, On the measurability of conditional expectations

Tom (Roy Thomas Jr.) Jacob, Matrix transformations involving simple sequence

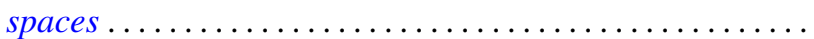

A. Katsaras, Continuous linear maps positive on increasing continuous

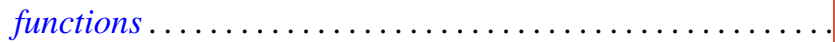

Kenneth Kunen and Judith Roitman, Attaining the spread at cardinals of cofinality

Lawrence Louis Larmore and Robert David Rigdon, Enumerating normal bundles

of immersions and embeddings of projective spaces ...... . .

Ch. G. Philos and V. A. Staïkos, Asymptotic properties of nonoscillatory solutions of differential equations with deviating argument .

Peter Michael Rosenthal and Ahmed Ramzy Sourour, On operator algebras containing cyclic Boolean algebras...

Polychronis Strantzalos, Strikt fast gleichgradig-stetige und eigentliche

Aktionen ...

Glenn Francis Webb, Exponential representation of solutions to an abstract

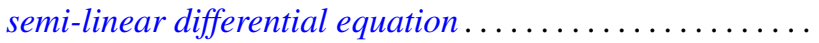

